$01.5 ; 11$

\title{
Многопользовательский доступ в системах относительной передачи информации на основе хаотических радиоимпульсов
}

\author{
(C) А.С. Дмитриев, Т.И. Мохсени, К.М. Сьерра-Теран \\ Институт радиотехники и электроники им. В.А. Котельникова РАН, Москва, Россия \\ E-mail: chaos@cplire.ru
}

Поступило в Редакцию 27 марта 2020 г.

В окончательной редакции 27 марта 2020г.

Принято к публикации 6 апреля 2020г.

\begin{abstract}
Показана возможность организации многопользовательского доступа на основе относительной схемы передачи, использующей в качестве носителя информации хаотические радиоимпульсы. Получены теоретические оценки максимального количества абонентов и информационной емкости многопользовательской системы. Проведено компьютерное моделирование, подтверждающее справедливость полученных теоретических результатов.
\end{abstract}

Ключевые слова: передача информации, хаотические радиоимпульсы, многопользовательский доступ.

DOI: 10.21883/PJTF.2020.13.49592.18323

Относительные методы передачи информации с широкополосными аналоговыми шумовыми (шумоподобными) сигналами исследуются начиная с 50-х годов $\mathrm{XX}$ века [1-3]. Однако долгое время развитию этих методов препятствовали сложности, связанные с формированием самих аналоговых шумовых сигналов. Ситуация стала меняться с появлением и разработкой принципов формирования шумоподобных сигналов радио- и СВЧ-диапазона на основе динамического хаоса.

Первая относительная схема передачи приемопередатчиков с хаотическими носителями информации DCSK (differential chaotic shift keying) была предложена в [4-6]. Идея DCSK заключается в том, что для передачи бита длительностью $T$ используется последовательная передача пары одинаковых фрагментов хаотического сигнала длительностью $T / 2$ каждый (для передачи „1“") или пары хаотического сигнала и ему антиподального сигнала тех же длительностей (для передачи „,“

B схеме DCSK используются широкополосные линии задержки на времена, равные $T / 2$. Их трудно реализовать в малогабаритном исполнении, что серьезно ограничивает практическую применимость схемы. Однако схема DCSK популярна при теоретических исследованиях. Так, например, после введения схемы DCSK как самими авторами, так и их последователями предлагались ее модифицированные варианты, в том числе с целью увеличения скорости передачи [7], повышения устойчивости в многолучевых каналах [8] и организации множественного доступа [9].

Недавно в [10-13] была предложена альтернативная схема относительной передачи информации на основе хаотических радиоимпульсов - $\mathrm{DC}^{2}$ (direct chaotic differential communication) (рис. 1), в которой отсутствует проблема с большими длительностями задержек. В этой схеме длительность задержек в приемнике и передатчике в отличие от схемы DCSK определяется не длительностью бита, а временем спадания функции автокорреляции $\tau$ хаотического сигнала. По порядку величины $\tau=1 / \Delta F$, где $\Delta F$ - полоса хаотического сигнала. Если, например, полоса хаотического сигнала будет равна $1 \mathrm{GHz}$, то время автокорреляции $\sim 1 \mathrm{~ns}$, а расстояние, которое за это время проходит электромагнитная волна в свободном пространстве, составит $0.3 \mathrm{~m}$. В этом случае в качестве линии задержки может быть использован, например, высокочастотный кабель длиной около $0.2 \mathrm{~m}$.

Цель настоящей работы - показать, что беспроводная схема передачи $\mathrm{DC}^{2}$ может быть эффективно использована не только в режиме передачи „точка-точка“ в одной паре передатчик-приемник, но и для организации одновременной и независимой работы нескольких пар приемников и передатчиков, находящихся в одной и той же области физического пространства.

$\mathrm{B}$ схеме $\mathrm{DC}^{2}$ для передачи информации используются хаотические радиоимпульсы с большим коэффициентом процессинга и защитными интервалами между импульсами. Введем следующие обозначения: $T_{i}$ - длительность хаотического радиоимпульса, $T_{b}$ - длительность бита, $L=T_{b} / T_{i}-$ скважность, $R-$ скорость передачи (в bit), $W$ - полоса сигнала, $K=W /(R L)$ - коэффициент процессинга для хаотического радиоимпульса, $\mathrm{SNR}$ - отношение сигнал/шум, $E_{b} / N_{0}=K \cdot \mathrm{SNR}$ отношение энергии, приходящейся на бит передаваемой информации, к спектральной плотности шума в полосе сигнала.

Особенностью схемы $\mathrm{DC}^{2}$ является наличие внутренних собственных шумов (внутренней интерференции) при передаче информации. Будем обозначать спектральную плотность этих шумов через $N_{i}$. На вход приемника кроме полезного сигнала поступают три „шумовые“ компоненты [10]. Интенсивность каждой из компонент шума такая же, как у полезного сигнала. Наличие этих 
шумовых компонент при отсутствии внешних шумов и применении антиподальной модуляции дает отношение сигнал/шум $\mathrm{SNR}=2 / 3$ или $\mathrm{SNR}_{\mathrm{dB}}=-1.75 \mathrm{~dB}$. Обычно энергетический потенциал канала связи определяется через минимальное требуемое значение отношения $E_{b} / N_{0}$, выраженное в $\mathrm{dB}$. В нашем случае при отсутствии внешних шумов $N_{0}=N_{i}$, и

$$
E_{b} / N_{0, \mathrm{~dB}}=E_{b} / N_{i, \mathrm{~dB}}=\mathrm{SNR}_{\mathrm{dB}}+K_{\mathrm{dB}} .
$$

Из (1) следует, что получение требуемого значения отношения $E_{b} / N_{i}$ при любом значении SNR может быть обеспечено за счет соответствующего выбора $K$. Например, в случае антиподальной модуляции вероятность ошибки $10^{-3}$ достигается при $E_{b} / N_{i}=10 \mathrm{~dB}$. Поэтому компенсация внутренних шумов системы обеспечивается при $K_{\mathrm{dB}}>11.75 \mathrm{~dB}$.

Пока рассматривались характеристики схемы без воздействия внешних шумов. Однако при достаточно большом процессинге сигнала система будет оставаться работоспособной даже при значительном уровне внешних шумов (SNR). В качестве этих внешних шумов могут, в частности, выступать сигналы других хаотических передатчиков, работающих в том же диапазоне частот. Это свойство схемы может быть положено в основу организации работы системы связи с множественным доступом.

Действительно, пусть имеется $M$ пар передатчиков и приемников (рис. 2). Все передатчики одновременно излучают, вообще говоря, разные сигналы, которые попадают как к своим приемникам в качестве полезного сигнала (точнее, часть сигнала, попадающего в „свой“ приемник, является полезной, а часть представляет собой собственный шум системы), так и в „чужие“ приемники в качестве шумового (интерферирующего) сигнала.

Для получения теоретической оценки будем считать, что во всех $M$ передатчиках используются генераторы хаоса с одинаковыми статистическими и спектральными характеристиками выходного сигнала, время автокорреляции хаотического сигнала равно $\tau_{0}$, длительности импульсов во всех парах приемопередатчиков равны. Времена задержек в $j$-й паре приемопередатчиков $\tau_{j}=j \tau_{0}$, $j=1,2,3, \ldots, M$. Такой набор задержек обеспечивает отсутствие корреляции своего сигнала в любом из $M$ приемников с чужими сигналами.

Покажем, что рассматриваемая схема относительной передачи с использованием хаотических радиоимпульсов позволяет реализовать множественный доступ, т.е. одновременную работу в эфире нескольких пар приемопередатчиков, в которых используются в том числе одинаковые генераторы хаоса.

Поскольку схема $\mathrm{DC}^{2}$ использует для извлечения полезного сигнала автокорреляционные свойства хаотического радиоимпульса, можно предположить, что применение процедуры корреляции будет также эффективно для выделения полезного сигнала пользователя на фоне

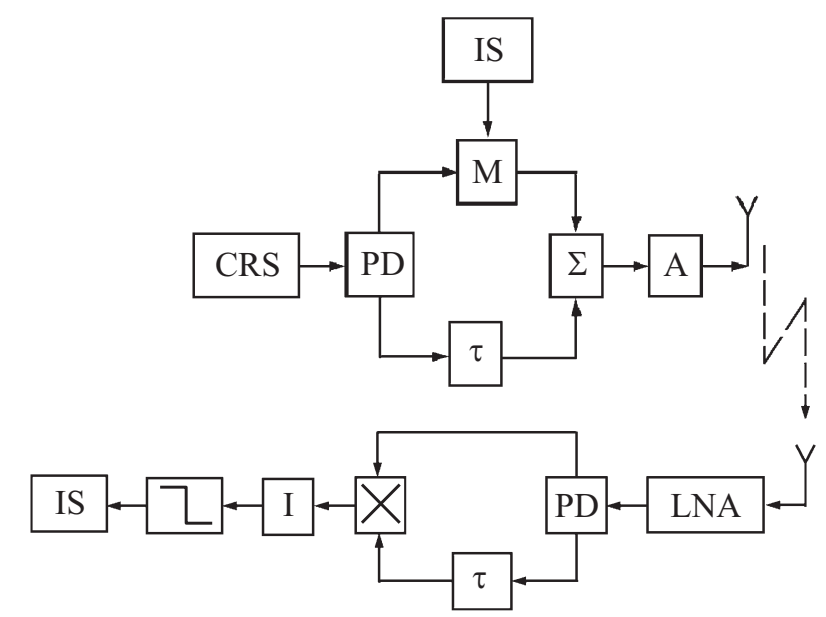

Pис. 1. Схема передачи: CRS - источник хаотических радиоимпульсов, PD - делитель, IS - информационная последовательность, М - модулятор, $\tau$ - задержка, $\sum-$ сумматор, A - усилитель, LNA - малошумящий усилитель, I - интегратор, крестиком обозначен перемножитель, знаком $乙-$ пороговое устройство.

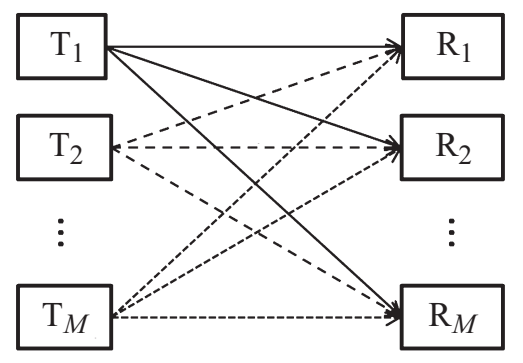

Рис. 2. Многопользовательская относительная система связи с $M$ парами передатчиков $\left(\mathrm{T}_{j}\right)$ и приемников $\left(\mathrm{R}_{j}\right)$.

сигналов других пользователей, рассматриваемых как внешний шум. Ситуация в этом случае будет подобна ситуации с выделением полезных сигналов в системах CDMA [14].

Будем считать, что в многопользовательской системе с $M$ парами приемопередатчиков применяется соответствующее управление мощностью излучения, так что сигналы, приходящие в данный $j$-й приемник от всех других устройств, имеют один и тот же уровень мощности $p$. Каждый приемник обрабатывает приходящий от своего передатчика сигнал на уровне мощности $p$, а $M-1$ посторонних сигналов интерферируют с ним, и каждый из этих сигналов также имеет уровень мощности $p$.

Отношение сигнал/интерференционная помеха на входе $j$-го приемника будет равно

$\mathrm{SNR}_{j}=(2 / 4) p /[(3 / 4) p+(M-1) p]=2 /[3+4(M-1)]$.

Из (2) видно, как максимально возможное число пользователей в сети связано с минимальным требуемым значением $\mathrm{SNR}_{j}$ на пользователя. Для схем связи с большим коэффициентом процессинга $K$ удобно перейти 

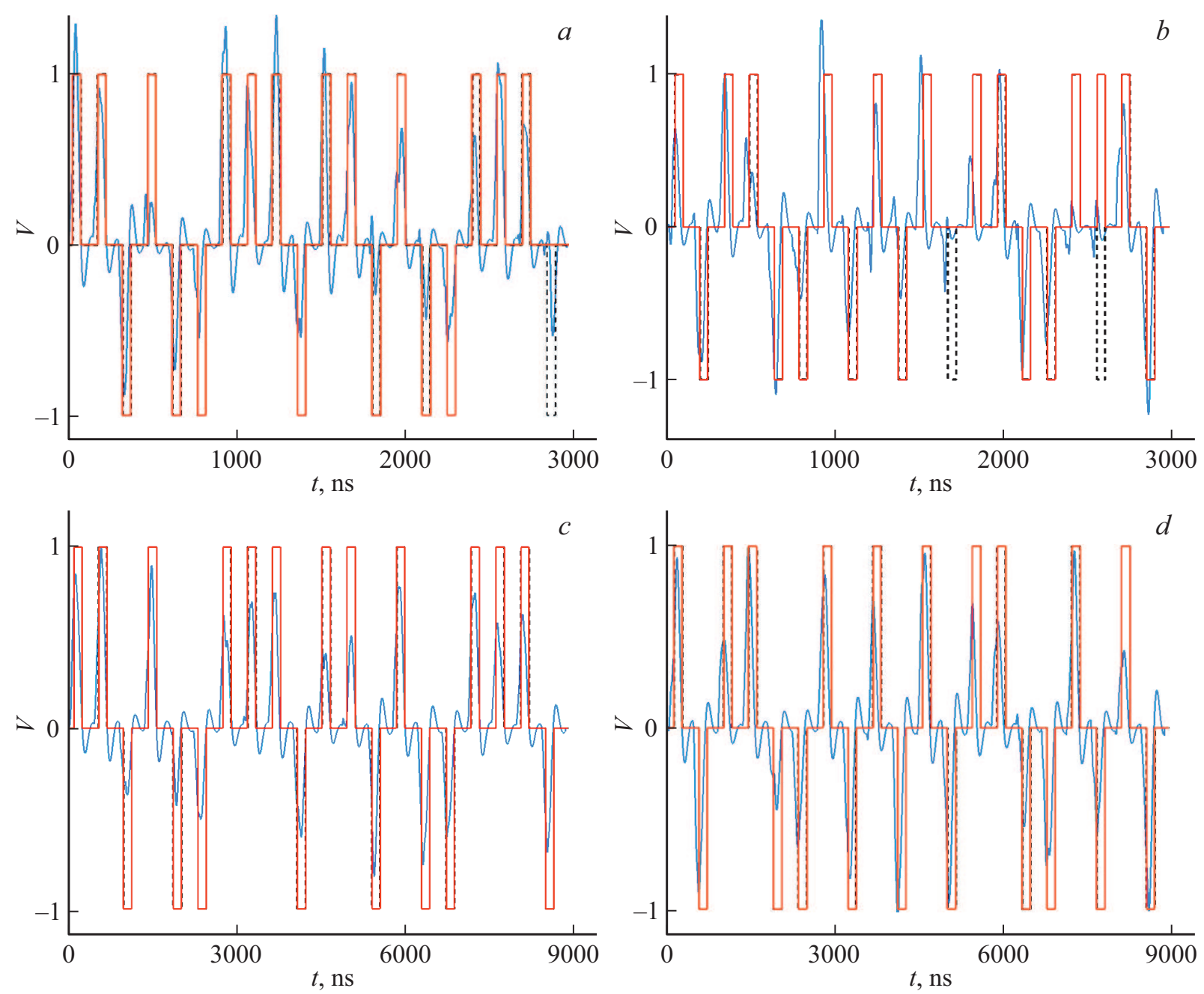

Рис. 3. Выходные сигналы в приемниках $1(a, c)$ и $4(b, d)$ при коэффициентах процессинга $100(a, b)$ и $300(c, d)$. Сплошные кривые - сигналы на выходах интеграторов. Прямоугольники - сигналы на выходах пороговых устройств. Прямоугольники со сплошной границей и пунктирной границей отвечают правильно и ошибочно принятым битам соответственно.

Зависимость числа пользователей $M$ и скорости передачи $R$ в системе от коэффициента процессинга $K$ при вероятности ошибок $10^{-3}$ и полосе сигнала $2 \mathrm{GHz}$

\begin{tabular}{c|c|c|c|c|c|c|c|c|c|c}
\hline \multirow{2}{*}{ Параметр } & \multicolumn{10}{c}{$K$} \\
\cline { 2 - 11 } & 15 & 100 & 150 & 200 & 300 & 400 & 800 & 1000 & 2000 & 4000 \\
\hline$M$ & 1 & 2 & 3 & 4 & 6 & 7 & 11 & 12 & 20 & 31 \\
$R$, Mbps & 64 & 10 & 6.6 & 5.0 & 3.3 & 2.5 & 1.25 & 1.0 & 0.5 & 0.25
\end{tabular}

от соотношения (2) к соотношению, связывающему максимальное число пользователей с $E_{b} / N_{i}$. Поскольку $E_{b} / N_{i}=K \cdot \mathrm{SNR}$, из (2) следует, что

$$
E_{b} / N_{i}=2 K /[3+4(M-1)]
$$

Если присутствует дополнительный мешающий внешний шум $\eta$, включающий интерференционный, не связанный с приемопередатчиками системы шум и тепловой шум, то при вводе его в рассмотрение уравнение (3) принимает вид

$$
E_{b} / N_{i}=2 K /[3+4(M-1)+4 \eta / p] .
$$

Это означает, что максимально возможное число пользователей в ячейке будет равно

$$
M=1 / 4\left[1+2 K /\left(E_{b} / N_{i}\right)\right]-\eta / p .
$$

Минимальное (требуемое для обеспечения заданной вероятности ошибки) значение отношения $E_{b} / N_{i}$ в схеме $\mathrm{DC}^{2}$ нелинейно зависит от процессинга. Оценка этой 
зависимости на основе аналитических соотношений, полученных в [10-12], приведена в таблице.

Для практического подтверждения возможности организации многопользовательского доступа на основе схемы передачи $\mathrm{DC}^{2}$ и проверки полученных теоретических оценок было проведено компьютерное моделирование. При этом задержки в приемопередатчиках подбирались таким образом, чтобы исключить возможность относительного корреляционного приема „чужих“ сигналов. Это достигалось за счет того, что в передатчиках использовались сигналы с одинаковым временем автокорреляции $\tau$, длины импульсов и защитных (межимпульсных) интервалов были одинаковы, а линии задержек у приемников и передатчиков в парах с номерами $1,2, \ldots, M$ были равны соответственно $\tau, 2 \tau, \ldots, M \tau$. Моделирование в целом дало хорошее совпадение с оценками, приведенными в таблице.

В качестве примера приведем результаты для $M=4$. Согласно данным таблицы, вероятность ошибки $p=10^{-3}$ достигается при коэффициенте процессинга $K>200$. Расчеты проводились для коэффициентов процессинга 100, 200 и 300. При $K=100$ и длительности информационной последовательности 1000 bit в каждом из каналов обнаруживалось более 10 ошибок (рис. 3, $a, b)$. При $K=200$ для той же длины последовательности наблюдались единичные ошибки. При моделировании с $K=300$ ошибки для последовательности длиной 1000 bit не наблюдались (рис. 3,c,d).

Таким образом, как теоретические оценки, так и результаты моделирования подтверждают возможность использования прямохаотической схемы относительной передачи $\mathrm{DC}^{2}$ в многопользовательских системах сверхширокополосной беспроводной связи.

\section{Конфликт интересов}

Авторы заявляют, что у них нет конфликта интересов.

\section{Список литературы}

[1] Петрович H.T., Размахнин М.К. Системы связи с шумоподобными сигналами. М.: Сов. радио, 1969. $232 \mathrm{c.}$

[2] Варакин Л.Е. Системы связи с шумоподобными сигналами. М.: Радио и связь, $1985.384 \mathrm{c.}$

[3] Петрович Н.T. Относительные методы передачи информации. М.: Книга-М, 2003. $111 \mathrm{c.}$

[4] Kolumban G., Kennedy M. // IEEE Trans. Circuits Syst. I. 1997. V. 44. N 10. P. 927-936.

[5] Kolumban G., Vizvari B., Schwarz W., Abel A. // Proc. NDES'96. Seville, Spain, 1996. P. 87-92.

[6] Kolumban G., Kennedy M.P., Jako Z., Kis G. // Proc. IEEE. 2002. V. 90. N 5. P. 711-732.

[7] Kaddoum G., Gagnon F. // IEEE Trans. Circuits Syst. II. 2012. V. 59. N 7. P. $448-452$.

[8] Kennedy M.P., Kolumban G., Kis G., Jako Z. // IEEE Trans. Circuits Syst. I. 2000. V. 47. N 12. P. 1702-1711.

[9] Lau F.C.M., Yip M.M., Tse C.K., Hau S.F. // IEEE Trans. Circuits Syst. I. 2002. V. 49. N 1. P. 96-104.
[10] Дмитриев А.С., Мохсени Т.И., Сьерра-Теран К.М. // Радиотехника и электроника. 2018. Т. 63. № 10. С. 1074 1082.

[11] Дмитриев А.С., Мохсени Т.И., Сьерра-Теран К.М. // Прикладная нелинейная динамика. 2018. Т. 26. № 4. С. 59 74.

[12] Dmitriev A.S., Mokhseni T.I., Sierra-Teran C.M. // Nonlinear Phenom. Complex Syst. 2018. V. 21. N 3. P. 237-246.

[13] Процессы передачи и обработки информации в системах со сложной динамикой / Под ред. А.С. Дмитриева, Е.В. Ефремовой. М.: Техносфера, 2019. $319 \mathrm{c}$.

[14] Шахнович И.В. Современные технологии беспроводной связи. М.: Техносфера, 2006. 287 с. 